\title{
SPECT in the localisation of extratemporal and temporal seizure foci
}

\author{
Mark R Newton, Samuel F Berkovic, Mark C Austin, Christopher C Rowe, \\ W John McKay, Peter F Bladin
}

\begin{abstract}
The yield of ictal, postictal, and interictal SPECT was compared in the localisation of seizure foci in 177 patients with partial epilepsy. In 119 patients with known unilateral temporal lobe epilepsy ictal SPECT ( $97 \%$ correct localisation) was superior to postictal SPECT $(71 \%$ correct), which was better than interictal studies (48\% correct). Similarly, in cases of known or suspected extratemporal epilepsy the yield of ictal SPECT studies was high (92\%). By contrast, the yield of postictal studies was much lower $(46 \%)$ and usually only very early postictal studies were diagnostic. Interictal SPECT was of little value. The accuracy of ictal SPECT in localising temporal lobe seizures is now well established. Extratemporal seizures are often brief and difficult to localise. This report shows that ictal SPECT also has a high diagnostic yield in a wide range of extratemporal epilepsies. The brevity of many extratemporal seizures means that true ictal SPECT examinations can be difficult to achieve, but the high diagnostic yield justifies the special organisational effort needed to obtain such studies.
\end{abstract}

$(\mathcal{F}$ Neurol Neurosurg Psychiatry 1995;59:26-30)

Department of

Neurology

M R Newton

$S$ F Berkovic

C C Rowe

P F Bladin

Department of Nuclear Medicine, Austin Hospital, Melbourne, Victoria, Australia

M R Newton

M C Austin

C C Rowe

W J McKay

Department of

Medicine, University

of Melbourne (Austin

Hospital), Victoria,

Australia

M R Newton

S F Berkovic

P F Bladin

Correspondence to:

Dr Samuel F Berkovic,

Department of Neurology,

Austin Hospital, Heidelberg

(Melbourne) Victoria 3084,

Australia.

Received 12 January 1994

and in revised form

3 January 1995 .

Accepted 10 February 1995 present a greater challenge for erative localisation. In some cases a discrete photon emission computed tomography (SPECT), ${ }^{4-13}$ without resorting to invasive intracranial recordings.

Patients with extratemporal epilepsies and temporal lobe epilepsies with unusual seizure
Surgical treatment of epilepsy is most succes confusion. ${ }^{2}$ Preoperative localisation of the focus in nearly all such patients can now achieved with clinical assessment, extracranial ictal EEG recordings, MRI, and functional lesion on MRI or a clearcut focal onset on ictal EEG will provide definitive localising information, but often localisation is difficult and requires intracranial EEG recordings. Intracranial recordings may not always be diagnostic, and even when the focus is thought to be localised, the surgical results are inferior to those of temporal lobe surgery. ${ }^{14} 15$ Interictal PET in cases without structural lesions has yielded generally disappointing results. ${ }^{16}$

Here we examine the value of SPECT in 177 consecutive patients with refractory partial seizures studied with ictal or postictal SPECT. We summarise our previously published results ${ }^{571013}$ in cases of well characterised unilateral temporal lobe epilepsy, now totalling 119 (plus seven patients with bitemporal foci), and compare these with the findings in the remaining 51 patients with established or probable extratemporal foci, possible temporal lobe epilepsy with unusual seizure patterns, and cases with unsolved localisation. We sought strategies whereby SPECT could help seizure focus localisation in patients with these complicated and heterogeneous partial epilepsies.

\section{Methods}

PATIENTS

One hundred and seventy seven patients were investigated for refractory partial seizures between 1987 and 1991 in the Comprehensive Epilepsy Programme at the Austin Hospital. All patients underwent clinical evaluation, video-EEG monitoring of seizures, MRI, and neuropsychological assessment. In addition, SPECT was performed on all patients after ictal or postictal injection of the blood flow imaging agent technetium-99m hexamethylpropyleneamineoxime ( ${ }^{99 \mathrm{~m}}$ Tc-HMPAO) ${ }^{10}$

\section{SPECT STUDIES}

SPECT was performed after injection of ${ }_{99 m}$ Tc-HMPAO during (ictal study) or after a seizure (postictal study) on at least one occasion during video-EEG monitoring, which allowed precise timing of the ligand injection relative to end of seizure. The methods for rapid preparation of ${ }^{99 \mathrm{~m}} \mathrm{Tc}-\mathrm{HMPAO}$, ictal injection of tracer, SPECT data acquisition, and display have been previously described. ${ }^{10}$ Our previous experience of SPECT in temporal lobe seizures has shown that the ictal hyperperfusion of the anterior temporal lobe persists up to 60 seconds into the immediate 
Table 1 Percentage localisation accuracy of epileptic foci using interictal, postictal, and ictal SPECT in 119 cases of unilateral temporal lobe epilepsy

\begin{tabular}{|c|c|c|c|}
\hline SPECT Lateralisation ${ }^{\star}$ & $\begin{array}{l}\text { Interictal } \\
(n=119)\end{array}$ & $\begin{array}{l}\text { Postictal } \dagger \\
n=77)\end{array}$ & $\begin{array}{l}\text { Ictal } \\
n=51\end{array}$ \\
\hline $\begin{array}{l}\text { Correct } \\
\text { Inconclusive } \\
\text { Incorrect }\end{array}$ & $\begin{array}{l}48 \\
42 \\
10\end{array}$ & $\begin{array}{r}71 \\
25 \\
4\end{array}$ & $\begin{array}{r}97 \\
3 \\
0\end{array}$ \\
\hline
\end{tabular}

*The first 46 cases were interpreted independently by two blinded reviewers, and the next 73 cases by three blinded blinded reviewers, and the next 73 cases by three blinded
reviewers. Values shown are the percentage means for the reviewers. Valu
whole series.

77 studies in 77 patients of whom nine also had ictal studies. $\$ 51$ studies in 51 cases. 49 of 51 cases were correctly lateralised by all three independent blinded reviewers. One case that was not lateralised by any reviewer had a repeat ictal study that showed correct lateralisation. A second case was not lateralised by only one of three reviewers.

postictal period, so that very early postictal injection would produce an ictal pattern of regional blood flow. ${ }^{9}$ We therefore classified such studies of temporal lobe epilepsy as ictal, and injections after the 30 second postictal period were defined as postictal studies. Studies in the non-temporal seizure group were called postictal when isotope was injected at any time after end of seizure. These studies were compared with interictal SPECT which were made when the patients had been seizure free for 24 hours (or as many hours postictally as their seizure frequency allowed). Ligand was injected with the patients supine and silent, with eyes closed in a suite, with minimal background noise.

\section{DATA REVIEW}

The methods of analysis of the SPECT data for the unilateral temporal lobe series has been reported previously..$^{5-10}$ For the other patients with either atypical temporal or extratemporal seizures two of the authors made provisional localisations from all clinical, video-EEG, and MRI data before examining the SPECT scans. Each patient was classified on the basis of these data as either definitely or probably localised, or unlocalised. The SPECT scans were then visually assessed by two of us (MRN and SFB) and classified as localising or non-localising on the basis of the finding of focal hyperperfusion.

\section{Results}

One hundred and nineteen patients were found to have unilateral temporal lobe epilepsy, and seven had proved or suspected bilateral temporal foci. Fifty one were considered to have partial seizures that were either not of temporal origin or were atypical for

Table 2 Localisation information from interictal, postictal, and ictal SPECT in 51 cases of proved or probable extratemporal foci, possible temporal lobe epilepsy with unusual seizure patterns, and unlocalised cases

\begin{tabular}{lccc}
\hline & Interictal & Postictal & Ictal \\
& SPECT & SPECT & SPECT \\
\hline Parietal $(\mathrm{n}=5)$ & $0 \%(0 / 5)$ & $0 \%(0 / 1)$ & $100 \%(4 / 4)$ \\
Frontal $(\mathrm{n}=5)$ & $20 \%(1 / 5)$ & $33 \%(1 / 3)$ & $100 \%(3 / 3)$ \\
Occipital $(\mathrm{n}=2)$ & $50 \%(1 / 2)$ & $100 \%(1 / 1)$ & $100 \%(2 / 2)$ \\
Atypical temporal $(\mathrm{n}=9)$ & $55 \%(5 / 9)$ & $83 \%(5 / 6)$ & $86 \%(6 / 7)$ \\
Unlocalised $(\mathrm{n}=30)$ & $33 \%(10 / 30)$ & $38 \%(10 / 26)$ & $89 \%(8 / 9)$ \\
Total $(\mathrm{n}=51)$ & $33 \%$ & $46 \%$ & $92 \%$ \\
\hline
\end{tabular}

temporal lobe epilepsy. Their mean age was 29 (range 11-60) years and mean duration of seizures was 15 (range 1-49) years.

\section{TEMPORAL LOBE SEIZURES}

Table 1 summarises our previously published data on 119 patients with unilateral temporal lobe epilepsy. ${ }^{5-10}$ Interictal SPECT may show focal hypoperfusion in the region of the seizure focus, whereas the ictal SPECT studies showed pronounced hyperperfusion in the discharging anterior temporal lobe. By contrast, postictal SPECT was characterised by lateral temporal hypoperfusion with preservation of the mesial temporal perfusion on the side of seizure focus. Ictal SPECT was clearly superior to postictal SPECT, which was better than interictal SPECT in localising the focus (table 1).

An additional five patients had well defined independent bilateral temporal lobe foci. In each case when an electroclinical right sided seizure was studied with ictal or postictal SPECT, the SPECT localised to the right side. Similarly, electroclinical left sided seizures localised to the left side with ictal or postictal SPECT. A further two patients had complex partial seizures, clinically suggestive of temporal lobe origin, that could not be definitively localised on electrical grounds, but ictal and postictal SPECT studies suggested bitemporal foci.

\section{EXTRATEMPORAL AND ATYPICAL TEMPORAL}

\section{CASES}

Table 2 shows the SPECT data in this heterogeneous group of cases. The value of SPECT in temporal lobe seizures (table 1) was assessed by comparing SPECT localisation to rigorous localisation by other means. In the remaining cases (table 2) putative localisation was made in 21 cases, but localisation was not possible in 30 patients. Localising information from ictal SPECT was obtained in $92 \%$ of cases studied, compared with $46 \%$ of postictal and $33 \%$ of interictal studies (table 2 ).

\section{PARIETAL LOBE SEIZURES}

Seizures arose from a parietal focus in five patients (table 2). The typical clinical pattern was a hemisensory aura followed by a sudden motor disturbance of one or more limbs, sometimes without alteration of consciousness. The most useful lateralising feature in four patients was the history of a unilateral sensory aura.

Although the ictal EEG tracings were unhelpful because of artefact, interictal EEG provided localising data in four patients. Magnetic resonance imaging showed a focal abnormality (cortical dysplasia) in only one case. The brevity of these seizures provided a difficult challenge for ictal SPECT study as preparation and injection of the ${ }^{99 \mathrm{~m}} \mathrm{Tc}-$ HMPAO usually requires at least $30 \mathrm{sec}-$ onds. ${ }^{10}$ Despite this, ictal injections were achieved and discrete regions of obvious hyperperfusion were seen in four of the SPECT studies (figure) providing clear evidence of the site of seizure origin. The site of 
Ictal SPECT study in a patient with seizures arising from the left parietal region due to focal cortical dysplasia. There is pronounced hyperperfusion in the left parietal area (arrow). Interictal SPECT was normal (not shown).

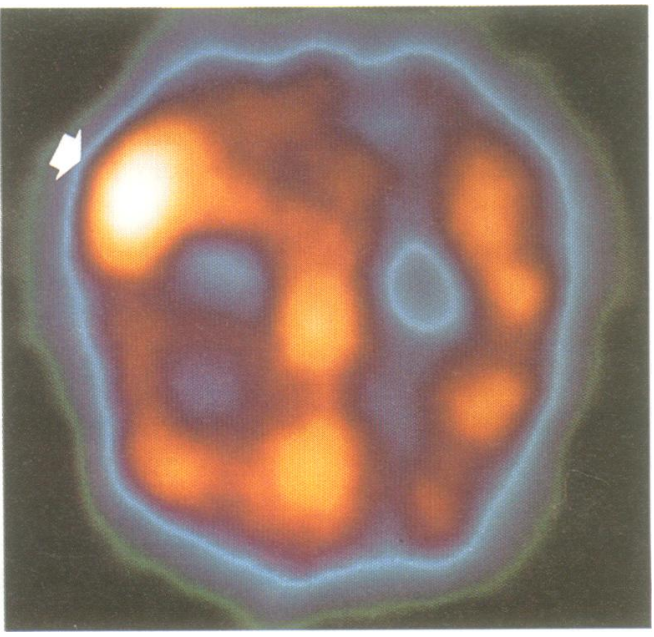

ictal SPECT hyperperfusion accorded with localisation deduced from other data in all four cases. The patterns of regional hyperperfusion were remarkable for the lack of widespread hypoperfusion distal to the discharging site that was commonly seen in the temporal ictal SPECT studies. ${ }^{89}$ The one postictal SPECT study did not demonstrate any asymmetry of perfusion, and all interictal SPECT studies were normal.

\section{FRONTAL LOBE SEIZURES}

Frontal lobe foci were found in five patients. Features that allowed confident lateralisation were lateralised auras (reported by three patients) and unilateral and dystonic or tonic posturing (in three) and clonic activity (in one). Interictal or ictal EEG (including two with intracranial electrodes) gave localising information in these five patients. Frontal atrophy was seen on MRI in three and one patient had central hemispheric dysplasia. The regional hyperperfusion of ictal SPECT confirmed the site of epileptic discharge in three, whereas postictal SPECT showed focal hypoperfusion in one and was unhelpful in another. Like the parietal studies, the ictal SPECT appearances were not associated with extensive distal hypoperfusion.

\section{OCCIPITAL LOBE SEIZURES}

Two patients had evidence of seizure foci involving the occipital lobe. Both had complex partial seizures with visual aura and lateralising ictal dystonia with normal MRI. In one patient surface ictal EEG showed temporooccipital discharge and ipsilateral hyperperfusion of the mesial occipital cortex during an uncommon seizure type consisting of staring and unresponsiveness. Study of his more typical complex partial seizures showed ictal EEG discharge from the right posterior quadrant and hyperperfusion of the lateral temporal and occipital cortex was seen with ictal SPECT. Electrocorticography at operation showed abundant epileptic activity over the posterior parietal and lateral occipital regions, so a resection of those areas was made (leaving him with a left inferior quadrantanopia). Pathology showed discrete areas of dysplastic cortex. The patient improved for six months but seizures returned. The patient was reinvestigated and a second ictal SPECT showed hyperperfusion in the parietal region superior to the resected margin so further cortical resection was undertaken after confirmation of the epileptic zone with ECG. Pathology again showed cortical dysplasia. The patient had been seizure free until three months after operation when a series of seizures were provoked by sleep deprivation and alcohol use. Freedom from seizures was restored with lifestyle correction.

Video-EEG recording of seizures from the other patient showed the origin of epileptic activity in the right posterior quadrant and ictal SPECT showed hyperperfusion throughout the right temporal and occipital lobes, maximal at the junction of the temporal, parietal, and occipital lobes. The patient's epilepsy responded to one of the new antiepileptic drugs before further investigation with intracranial EEG.

\section{ATYPICAL SEIZURES OF PROBABLE TEMPORAL} LOBE ORIGIN

Nine patients had seizures not typical of temporal lobe epilepsy yet EEG or other data suggested a temporal lobe origin. The ictal features of this group included hyperkinesis, unilateral clonic jerking, peculiar vocalisations with bilateral upper limb posturing, and intermittent giggling; MRI showed temporal lobe lesions in six patients; EEG suggested a unilateral temporal focus in six and another showed a temporo-occipital focus.

The patient with the temporo-occipital focus had a gliotic area in the left occipital lobe on MRI. Ictal scalp EEG revealed posterior temporal origin of seizures, confirmed with intracranial EEG study. Cortical resection of the temporo-occipital cortex, however, showed no specific pathology and seizures returned. Two preoperative ictal SPECT studies showed anterior mesial and lateral temporal hyperperfusion respectively, and a repeat ictal SPECT study postoperatively again showed anterior hyperperfusion. A standard anterior temporal lobectomy was then performed and the patient has had auras only at two years follow up.

Ictal SPECT was obtained in another six cases. Three showed unilateral temporal hyperperfusion that accorded with the side of the focus suggested by MRI, EEG, or both. Two showed symmetric bitemporal hyperperfusion on ictal SPECT; both had prominent vocalisation and bilateral upper limb posturing during their seizures, which began with bilateral temporal EEG discharge in both cases; MRI was normal in one and showed bilateral hippocampal sclerosis in the other. Another patient had ambiguous changes in his ictal scans. Postictal and interictal scans provided some lateralising information but were inferior to ictal studies (table 2).

\section{UNLOCALISED FOCAL SEIZURES}

The seizure focus was not clearly localised in 30 patients, although some lateralising or 
localising information was obtained from clinical, EEG, and MRI studies in certain cases. Clinically useful features in the seizures of this group were scarce, and the duration of seizures was often brief. There was a wide range of ictal manifestations, which included dystonic cruciate posturing of upper limbs, brief tonic posturing of one arm, giggling with incontinence, whooping and grimacing with dystonic posturing of either hand, and staring with immobility of all limbs.

Thirty nine SPECT peri-ictal scans were obtained in the 30 patients (table 2). There were 29 postictal studies in 26 patients, with a mean delay in injection of 3.2 minutes (range 15 seconds to 6.5 minutes), and 10 ictal SPECT studies in nine patients. Five patients underwent both postictal and ictal SPECT.

Ictal SPECT gave new diagnostic information in four patients, supported the clinical or EEG data in four, and was not helpful in one. Postictal scans provided novel information in three, supported the localisation in seven, and gave no useful data in 16 patients. Only the ictal SPECT showed focal changes in four of the five patients who had both ictal and postictal studies, but both SPECT studies were helpful in the remaining patient.

Overall, SPECT contributed corroborative localising data in 10 of the 30 patients and provided the only localising information for another five. The suspected foci were unilateral temporal (five), bitemporal (two), parasagittal (two), frontotemporal (two), orbitofrontal (two), parietal (one), and multifocal (one).

\section{Discussion}

The techniques of EEG remain the principal investigation for localising seizure foci or confirming the site suggested by clinical features. Uncontaminated scalp EEG recordings, however, cannot often be obtained in patients with pronounced ictal motor activity, as seen in many of the seizures studied here. Furthermore, intracranial electrode studies should only be used when a specific region or regions are suspected as being epileptogenic, as these invasive studies cannot explore the whole cortex. When clinical features and EEG are not localising, and structural neuroimaging studies are normal, there is left little diagnostic scope for the further investigation of these patients. It is in this context that functional imaging with SPECT will be of great value. The relative simplicity of ligand injection and data acquisition allows the technique to be exploited in most epilepsy monitoring programmes.

The clinical value and reliability of SPECT in the identification of temporal lobe foci has only recently been established. ${ }^{13}$ Ictal SPECT study yields a greater proportion of correct lateralising data than either postictal or interictal SPECT (table 1). Although inferior to ictal SPECT, postictal SPECT in temporal lobe seizures remains a more powerful and reliable study than interictal SPECT

The value of SPECT in the localisation of extratemporal foci is not clear. Reports of
${ }^{99 \mathrm{~m} T c-H M P A O ~ S P E C T}$ in this domain of partial epilepsy are few. ${ }^{617-19}$ The abruptness of onset, brevity, and pronounced motor activity of many of the seizure types militate against ready capture with the ligand. This contrasts with seizures arising from the temporal lobe, which are often heralded by an aura, last more than a minute, and rarely contain violent movements thereby making ligand injection easier. Despite these inherent difficulties, we obtained SPECT data from many patients with suspected extratemporal foci over a five year period to evaluate the clinical utility of SPECT in this subgroup of refractory focal epilepsy.

The results of the 51 cases as a whole (table 2) show the superiority of ictal SPECT over postictal and interictal studies. Not only was the yield of diagnostic studies higher in a quantitative sense, but the studies were also easier to interpret. These overall results were also applicable to the individual subgroups; ictal SPECT accurately localised all parietal, frontal, and occipital cases where a confident electroclinical diagnosis had been made. Postictal study was less rewarding, however, and interictal study the least (table 2). Ictal study also suggested a temporal site of origin in $86 \%$ of patients with seizures atypical of temporal lobe epilepsy in whom there was some other evidence for a temporal focus despite the unusual clinical features.

Ictal SPECT was also helpful in cases unlocalised on clinical or EEG criteria. This included four patients in whom SPECT provided the only useful data that can now direct further investigation, if clinically appropriate, with invasive EEG studies. This clearly represents a great advance in the investigation of this very difficult subgroup of refractory focal epilepsies.

Although ictal SPECT was the more rewarding study in the unlocalised group, postictal SPECT studies were the most commonly performed. Two factors accounted for this. Half of these patients were investigated before the refinement of ${ }^{99 \mathrm{~m} T \mathrm{~T}-H M P A O}$ preparation that made the ligand ready for injection in 30 seconds, so that injections before this important development were always postictal. Also, the combined delays of seizure alert, summons of the person to inject, and ligand preparation precluded capture of the ictal phase in seizures of very brief duration (for example, less than 45 seconds).

The excellent localising information derived from ictal SPECT in the unlocalised group was expected with capture of the regional hyperperfusion that accompanies the focal cortical discharge. There was, however, a discrepancy between the localisation rates of their postictal SPECT and those of the temporal series. The relatively poor performance of the postictal SPECT in the unlocalised group compared with the temporal series prompted further analysis.

Postictal SPECT provided localising data in only 10 of the 26 patients of the unlocalised group studied in that condition. The mean delay in injection was $4 \cdot 1$ (range $1-15$ ) minutes and a temporal focus was identified in 
eight of the patients. Frontotemporal and central parasagittal foci were found in the other two patients who were injected at 16 seconds and 20 seconds postictally. Postictal SPECT was of no value in the other 16 patients where the mean delay in injection was $3 \cdot 1$ (range $0 \cdot 25-0 \cdot 65$ ) minutes. Three of these patients were proved to have extratemporal foci on ictal SPECT study.

Thus despite similar delays in ligand injection, the diagnostic yield of the SPECT in the patients with extratemporal focus was different from the temporal group. This suggests that postictal perfusion patterns in temporal lobe seizures and in seizures of extratemporal origin evolve at different rates. It is likely that the sequence of ictal to postictal changes in regional cortical perfusion in extratemporal seizures evolves over a much shorter time frame than in temporal lobe seizures. In temporal lobe seizures, the characteristic postictal perfusion patterns are established in the second postictal minute ${ }^{9}$ and persist for up to 15 minutes before returning to the interictal state. $^{7}$

In addition, the topography of cortical blood flow distribution after extratemporal lobe seizures seemed to differ from that of temporal lobe seizures. The examples of postictal hypoperfusion shown by SPECT in extratemporal lobe seizures were restricted to the immediate region of the seizure focus. By contrast, temporal lobe seizures are usually followed by the characteristic widespread ipsilateral cortical depression of blood flow, often sparing the mesial temporal region. ${ }^{579}$ These findings likely reflect variations in the underlying physiology of regional cortical blood flow that accompany seizures arising from different sites, and have important implications for the use of SPECT. The lower yield of diagnostic information from postictal SPECT in suspected extratemporal seizures demands that the ligand should be injected during the seizure to exploit the easily interpretable ictal hyperperfusion.

MRN was supported by a Ciba-Geigy (Australia) Epilepsy Fellowship. MRN, CCR, and SFB were supported by the National Health and Medical Research Council of Australia.
1 Rasmussen TB. Surgical treatment of complex partial seizures: results, lessons and problems. Epilepsia 1983 24(suppl 1):65-76.

2 Weiser HG, Engel J Jr, Williamson PD, Babb TL, Gloor $P$. Surgically remediable temporal lobe syndromes. In Engel J Jr, ed. Surgical treatment of the epilepsies. 2nd ed. New York: Raven Press, 1993:49-63.

3 Engel J Jr, Henry TR, Risinger MW, et al. Pre-surgical evaluation for partial epilepsy: relative contributions of
chronic depth-electrode recordings versus FDG-PET and scalp-sphenoidal ictal EEG. Neurology 1990;40: and scalp-7.

4 Lee BI, Markand ON, Siddiqui AR, et al. Single photon emission computed tomography (SPECT) brain imaging using HIPDM: intractable complex partial seizures. Neurology 1986;36:1471-7.

5 Rowe CC, Berkovic SF, Sia STB, et al. Localisation of epileptic foci with postictal single photon emission computed tomography. Ann Neurol 1989;26:660-8.

6 Stefan H, Bauer J, Feistel H, et al. Regional cerebral blood flow during focal seizures of temporal and frontocentral onset. Ann Neurol 1990;27:162-6.

7 Rowe CC, Berkovic SF, Austin MC, McKay WJ, Bladin PF. Patterns of postictal cerebral blood flow in temporal lobe epilepsy: qualitative and quantitative analysis. Neurology 1991;41:1096-103.

8 Newton MR, Berkovic SF, Austin MC, et al. Dystonia clinical lateralization, and regional cerebral blood flow in temporal lobe seizures. Neurology 1992;42:371-7.

9 Newton MR, Berkovic SF, Austin MC, Rowe CC, McKay WJ, Bladin PF. Postictal switch in blood flow distribution and temporal lobe seizures. $\mathcal{f}$ Neurol Neurosurg tion and temporal lobe
Psychiatry 1992;55:891-4.

10 Newton MR, Austin MC, Chan JG, McKay WJ, Rowe CC, Berkovic SF. Ictal SPECT using ${ }^{99}$ HMPAO: methods for rapid preparation and optimal deploymen of tracer during spontaneous seizures. $₹ \mathrm{Nucl} \mathrm{Med}$ 1993;34:666-70

11 Harvey AS, Bowe JM, Hopkins IJ, Shield LK, Cook DJ, Berkovic SF. Ictal ${ }^{99 m}$ Tc-HMPAO single photon emission computed tomography in children with temporal lobe epilepsy. Epilepsia 1993;34:869-77.

12 Duncan R, Patterson J, Roberts R, Hadley DM, Bone I. Ictal/postictal SPECT in the presurgical localisation of complex partial seizures. $\mathcal{F}$ Neurol Neurosurg Psychiatry complex partial

13 Berkovic SF, Newton MR, Chiron C, Dulac O. Single photon emission tomography. In: Engel J Jr, ed. Surgical treatment of the epilepsies. 2nd ed. New York: Raven Press, 1993:233-43.

14 Van Ness PC. Surgical outcome for neocortical (extra-hippocampal) focal epilepsy. In: Lüders HO, ed. Epilepsy surgery. New York: Raven Press, 1992:613-24.

15 Williamson PD, Van Ness PC, Weiser HG, Quesney LF Surgically remediable extratemporal syndromes. In Engel J Jr, ed. Surgical treatment of the epilepsies. 2nd ed. New York: Raven Press, 1993:65-76.

16 Henry TR, Sutherling WW, Engel J Jr, Risinger MW, Levesque MF, Mazziotta JC, Phelps ME. Interictal cerebral metabolism in partial epilepsies of neocortical oribral metabolism in partial epilep

17 Marks DA, Katz A, Hoffer P, Spencer SS. Localization of extratemporal epileptic foci during ictal single photon emission computed tomography. Ann Neurol 1992;31: 250-5.

18 Harvey AS, Hopkins IJ, Bowe JM, Cook DJ, Shield LK, Berkovic SF. Frontal lobe epilepsy: clinical seizure characteristics and localization with ictal ${ }^{99 \mathrm{~m} T \mathrm{~T}-\mathrm{HMPAO}}$ SPECT. Neurology 1993;43:1966-80.

19 Kuzniecky R, Mountz JM, Wheatley G, Morawetz R. Ictal single photon emission computed tomography demonstrates localized epileptogenesis in cortical dysplasia. Ann Neurol 1993;34:627-31. 